\title{
Proximal Urethra
}

National Cancer Institute

\section{Source}

National Cancer Institute. Proximal Urethra. NCI Thesaurus. Code C115335.

The part of the urethra that is closer to the bladder. 